\title{
Surface EMG Analysis of Biceps Brachii Fatigue During Isometric Contraction: A Preliminary Study
}

\author{
Yumeng $\mathrm{Li}^{*}$ \\ Department of Kinesiology, California State University, Chico, CA, USA \\ *Corresponding author: Yumeng Li, Department of Kinesiology, California State University, 400 West 1st \\ St., Chico, CA, USA, Tel: +1 7062552499; E-mail: yli41@csuchico.edu
}

Received Date: 26 September, 2017; Accepted Date: 20 November, 2017; Published Date: : 05 December, 2017

\begin{abstract}
There have been a lot of studies regarding muscle fatigue and fatigue mechanisms; however, there is still little research on reliable ways to measure muscle fatigue and determine how this information can be beneficial. Therefore, the purposes of the present study were (1) to examine the median and mean power frequency during isometric biceps brachii contraction (2) to determine whether a correlation existed between the two parameters in depicting muscle fatigue. Two male participants were tested during a biceps brachii fatigue test using a surface EMG system. The power spectrum of EMG data shifted towards the lower frequency during the muscle fatigue assessments. The mean power frequency decreased consistently during the fatigue test $(\mathrm{r}=-0.338, \mathrm{p}=0.008)$. However, the median power frequency was not correlated with time $(r=-0.128, p=0.329)$. In addition, these two parameters were highly correlated $(\mathrm{r}=0.825$, $\mathrm{p}=0.001)$. Therefore, we suggest that using both parameters to obtain more information would be beneficial for muscle fatigue studies.
\end{abstract}

\section{Introduction}

Muscle fatigue represents a complex phenomenon encompassing various causes, mechanics and forms of manifestation [1]. Many studies have been done to describe the phenomenon and mechanisms responsible for muscle fatigue [2-4]. Based on the previous studies, muscle fatigue can be defined as the activity-induced loss of the force-producing capability of the muscle [5]. Though it is not difficult to define muscle fatigue, determining and quantifying fatigue is another case. Cifrek et al., [1] stated that the simplest way to determine the onset of muscle fatigue was to measure time during which an individual was able to perform certain work, which was known as 'mechanical manifestation of muscle fatigue'. Compared to mechanical measures, Electromyography (EMG) analyses are more objective means and provide an early indication of the fatiguing process [6].
Previous studies attempted to use the amplitude analysis of the EMG signal as a measure of muscle fatigue $[7,8]$. The commonly used variables to analyze amplitude of EMG are rectified integration (iEMG) and Root Mean Square value (RMS). An increase in amplitude of the EMG was observed during muscle fatigue [7,8]. However, Winter [9] suggested the amplitude was just a reflection of the frequency shift. Moreover, the amplitude could vary with the type of electrode used, the placement of electrode, and the time-dependent properties of the conducting gels that were commonly used to interface the electrodes to skin [9].

The most commonly used parameters for fatigue analysis in recent studies were median power frequency and mean power frequency. The median power frequency is the frequency at which power density spectrum is divided into two regions with equal power, whereas the mean power frequency is the weighted average frequency in which each frequency component is weighted by its power [10]. Researchers suggested that a decrease in median and mean power frequency over time could depict muscle fatigue $[10,11]$.

There have been a lot of studies regarding muscle fatigue and fatigue mechanisms; however, there is still little research on detecting muscle fatigue and determining how this information can be beneficial. Moreover, it is still unclear which parameter of the power spectrum could depict fatigue more effectively. Therefore, the purposes of the present study were (1) to examine the median and mean power frequency during isometric biceps brachii contraction (2) to determine whether a correlation existed between the two parameters in depicting muscle fatigue. Based on current knowledge, the investigator made two hypotheses: (1) both the median and mean power frequency decrease during fatigue; (2) the two parameters were highly correlated.

\section{Methods}

\section{Participants}


Two healthy, recreationally active individuals (males, age $=25$ $\mathrm{yr}$ and $35 \mathrm{yr}$, mass $=66$ and $75 \mathrm{~kg}$, height $=1.71$ and $1.72 \mathrm{~m})$ voluntarily participated. Both participants were right-hand dominant and participated in a weight training program $(\sim 5 \mathrm{hr} / \mathrm{wk})$. Informed consent was obtained before data collection.

\section{Data collection}

A surface EMG system (Myopac ${ }^{\circledR}$, Run Technologies, Inc.) was used to measure muscle activation. The skin was shaved and cleaned with alcohol to reduce the skin impedance. Two bipolar silver-silver chloride surface electrodes (8-mm diameter) were attached on the muscle belly of the biceps brachii of the right arm in a direction parallel to the muscle fibers. The electrode placement locations were selected based on the previous study [12]. The inter-electrode distance was $2 \mathrm{~cm}$. The Common-Mode Rejection Ratio (CMRR) of the unit was $110 \mathrm{~dB}$. The electrode gain was set at 1000 . One extra electrode was placed on the left olecranon of the ulna as a reference (ground electrode). The surface EMG signal was collected at $1200 \mathrm{~Hz}$. There was no filtering applied by the hardware. All the EMG data were recorded and stored using the Vicon work station software (Vicon Motion System Ltd.).

The baseline EMG signal was collected for three seconds for each participant. The participants were in a standard anatomical position with relaxed arms. Then, the participants stood on a strength test machine (Polar ${ }^{\circledR}$ TriFit system, Gopher Sport.) and held the pulling bar with a $90^{\circ}$ elbow flexion angle (Figure 1). When the start signal was given by the investigator, the participant pulled the bar upward as hard as possible with only right arm elbow flexors for 5 seconds. As the bar did not move, the participant performed a Maximal Voluntary Isometric Contraction (MVIC). The MVIC load (a force measured during the MVIC test) was recorded. The participants took a 10-minute break after the MVIC tests. The participants sat on the chair and held a $60 \%$ of the MVIC load dumbbell with $90^{\circ}$ elbow flexion. The dumbbell weighed at $60 \%$ of the MVIC load was used because above this level of force output there was a greater recruitment of type two muscle fibers compared to lower levels of force output [13]. Type two muscle fibers have relatively low resistance to fatigue, therefore this force output design was easier to achieve muscle fatigue. The participants held the dumbbell and maintained the arm position for 30 seconds. A 30-second test duration was used because both participants demonstrated fatigue at 30 seconds based on preliminary testing.

\section{Data analysis}

The raw EMG was processed by the following steps using customized MATLAB (The MathWorks, Inc., Natick, MA, USA) programming codes: (1) adjust to the baseline: the baseline data was derived using the mean value of the baseline EMG trial, then the raw EMG data was deducted by this mean value; (2) filter with a band-pass $(10-250 \mathrm{~Hz})$ Butterworth filter (the cutoff frequency was determined based on power spectrum); (3) recti- fy the filtered EMG data; (4) apply the Fast Fourier Transform (FFT) to get the power spectrum. (5) Calculate median power frequency and mean power frequency within one-second time windows.

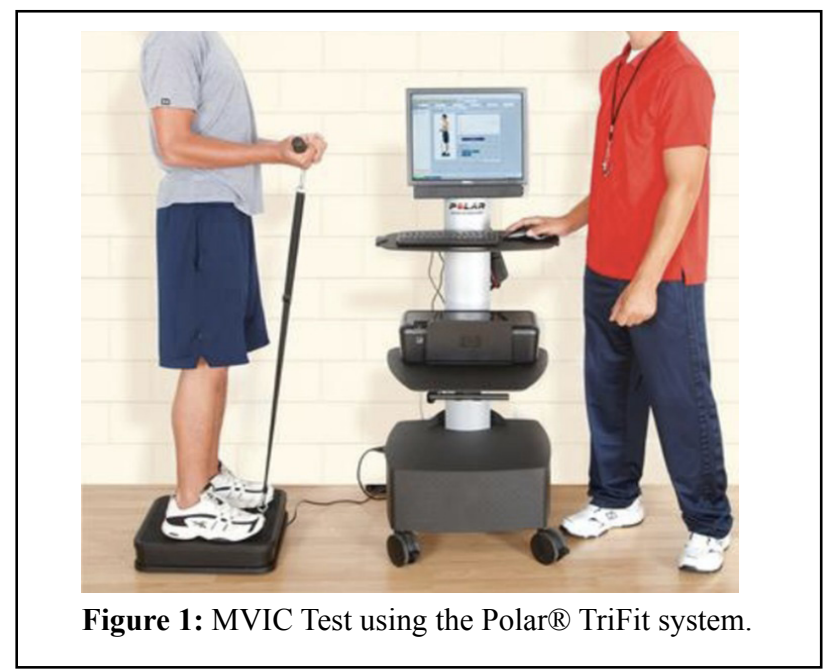

Pearson correlation was used to examine the correlation between median power frequency and time, between mean power frequency and time, and between median power frequency and mean power frequency. Participants' data were combined for statistical analyses. Statistical significance was set at $\mathrm{p}<0.05$. All statistical analyses were processed using SPSS (IBM Corp., Armonk, NY, USA).

\section{Results}

The median power frequency (Figure 2) was not significantly correlated with time $(\mathrm{r}=-0.128, \mathrm{p}=0.329)$. The mean power frequency was significantly correlated with time $(\mathrm{r}=-0.338, \mathrm{p}=$ 0.008 ), which indicated the mean power frequency reduced during the fatigue process (Figure 3). In addition, the median and mean power frequencies were highly correlated $(\mathrm{r}=0.825, \mathrm{p}=$ 0.001 ). The power spectrum was presented in figure 4 .

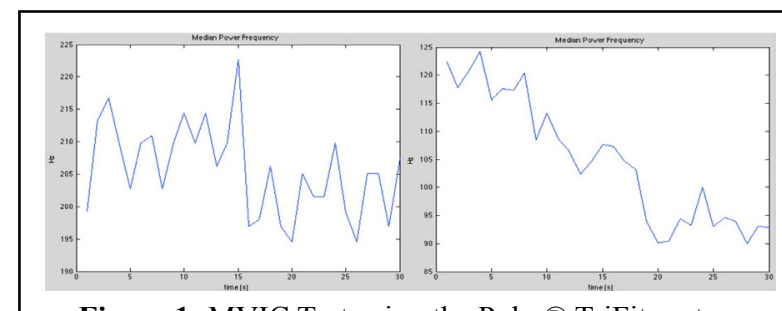

Figure 1: MVIC Test using the Polar ${ }^{\circledR}$ TriFit system.

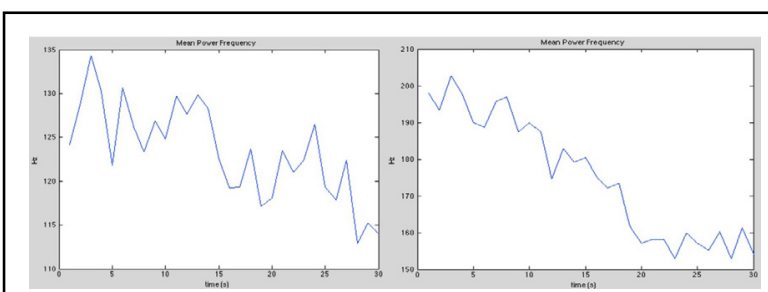

Figure 2: Median power frequency of the two participants during the fatigue test. 


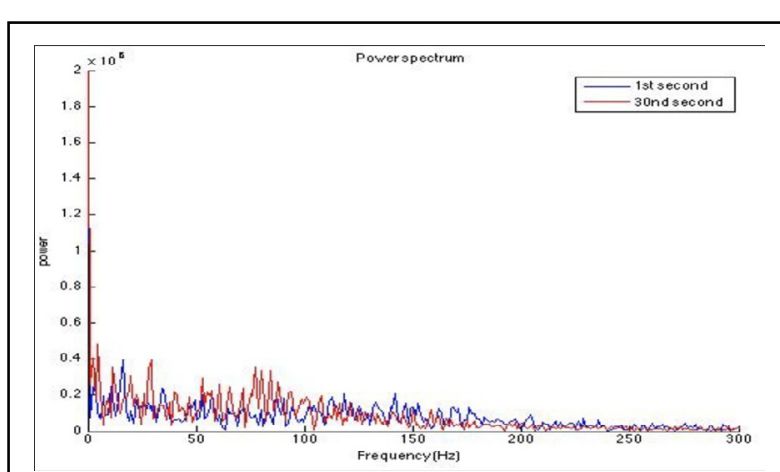

Figure 3: Mean power frequency of the two participants during the fatigue test.

\section{Discussion}

The power spectrum shifted towards the lower frequency during the muscle fatigue process. However, different than our hypothesis, the median power frequency was not significantly correlated with time. Therefore, in this case, the median power frequency did not successfully depict the muscle fatigue. One potential reason was the shape of the spectrum. Though fatigue occurred and the overall power spectrum shifted to the left (lower frequency), the median power frequency could still remain the same due to specific shapes of the spectrum [9]. Winter [9] also suggested that other related factors such as the quality of the EMG signal could also affect median power frequency.

As we hypothesized, the mean power frequency was significantly correlated with the time and reduced consistently over time. The mean power frequency was initially $\sim 165 \mathrm{~Hz}$ and reduced to $\sim 125 \mathrm{~Hz}$ at the end of test. The results were similar but slightly greater compared to those reported in previous studies $[12,14]$. The researchers measured mean power frequency following fatigue of the elbow flexors and reported that the mean frequency was $100-105 \mathrm{~Hz}$ prior to the fatiguing exercise and declined to $\sim 70 \mathrm{~Hz}$ after exercise. The discrepancy between the present study and previous studies $[12,14]$ was possibly due to the fatigue process (60\% MVIC load vs. 50\% MVIC load) [12], different muscles (biceps brachii vs. triceps brachii) [14] and small sample size in the present study.

Though the median and mean power frequency were highly correlated, using only one of them was probably not enough to detect fatigue effectively. As described previously, the median power frequency could depend on the shape of the spectrum, quality of the EMG signal and other related factors. However, we cannot suggest that one parameter is superior to the other. Stulen and De Luca [15] suggested that median power frequency provided a reliable, consistent and relatively unbiased estimate of a parameter of the spectrum that is related to the muscle fiber conduction velocity. Other researches preferred the mean frequency and demonstrated that the mean frequency was linearly related to the average conduction velocity of muscle fibers [12]. However, to our knowledge, there is no strong evidence to conclude that one parameter is always superior to the other one in all situations. Therefore, we suggest that using both of the parameters could be beneficial to detect muscle fatigue effectively.

The primary limitation in the present study is the small sample size. As a preliminary study, only two participants were enrolled. Due to relatively weak statistical power, we cannot make any convincing suggestions. Future studies should recruit more participants to increase the statistical power.

In conclusion, the power spectrum shifted towards the lower frequency during muscle fatigue process. The mean power frequency decreased consistently during the fatigue test. However, the median power frequency was not correlated with time. Even though these two parameters were highly correlated, using only one of them may not detect muscle fatigue effectively. Therefore, we suggest that using both parameters to obtain more information, which would be beneficial for muscle fatigue studies.

\section{Conflict of Interest Statement}

No author has any financial or personal relationship with other people or organizations that could inappropriately influence or bias his/her work.

\section{References}

1. Cifrek M, Medved V, Tonkovic S, Ostojic S (2009) Surface EMG based muscle fatigue evaluation in biomechanics. Clin Biomech (Bristol, Avon) 24: 327-340.

2. Allen DG, Westerblad $H$ (2001) Role of phosphate and calcium stores in muscle fatigue. J Physiol 536: 657-665.

3. Enoka RM, Duchateau J (2008) Muscle fatigue: what, why and how it influences muscle function. J Physiol 586: 11-23.

4. Kallenberg LA, Schulte E, Disselhorst-Klug C, Hermens HJ (2007) Myoelectric manifestations of fatigue at low contraction levels in subjects with and without chronic pain. J Electromyogr Kinesiol 17: 264-274.

5. Christie A, Snook EM, Kent-Braun JA (2011) Systematic review and meta-analysis of skeletal muscle fatigue in old age. Med Sci Sports Exerc 43: 568-577.

6. Izquierdo M, Gonzalez-Izal M, Navarro-Amezqueta I, Calbet JA, Ibañez J, et al. (2011) Effects of strength training on muscle fatigue mapping from surface EMG and blood metabolites. Med Sci Sports Exerc 43: 303-311.

7. Hagberg M (1981) Muscular endurance and surface electromyogram in isometric and dynamic exercise. J Appl Physiol Respir Environ Exerc Physiol 51: 1-7.

8. Maton B (1981) Human motor unit activity during the onset of muscle fatigue in submaximal isometric isotonic contraction. Eur J Appl Physiol Occup Physiol 46: 271-281.

9. Winter DA (1990) Biomechanics and motor control of human movement. 2ndedn, Wiley, Hoboken, NJ, USA.

10. Oberg T, Sandsjo L, Kadefors R (1994) Subjective and objective evaluation of shoulder muscle fatigue. Ergonomics 37: 1323-1333. 
11. Allison GT, Fujiwara T (2002) The relationship between EMG median frequency and low frequency band amplitude changes at different levels of muscle capacity. Clin Biomech (Bristol, Avon) 17 464-469.

12. Williams DM, Sharma S, Bilodeau M (2002) Neuromuscular fatigue of elbow flexor muscles of dominant and non-dominant arms in healthy humans. J Electromyogr Kinesiol 12: 287-294.

13. Minning S, Eliot CA, Uhl TL, Malone TR (2007) EMG analysis of shoulder muscle fatigue during resisted isometric shoulder elevation. J Electromyogr Kinesiol 17: 153-159.
14. Stirn I, Jarm T, Kapus VP, Strojnik V (2013) Evaluation of mean power spectral frequency of EMG during 100 metre crawl. Eur J Sport Sci 13: 164-173.

15. Stulen FB, DeLuca CJ (1981) Frequency parameters of the myoelectric signal as a measure of muscle conduction velocity. IEEE Trans Biomed Eng 28: 515-523. 